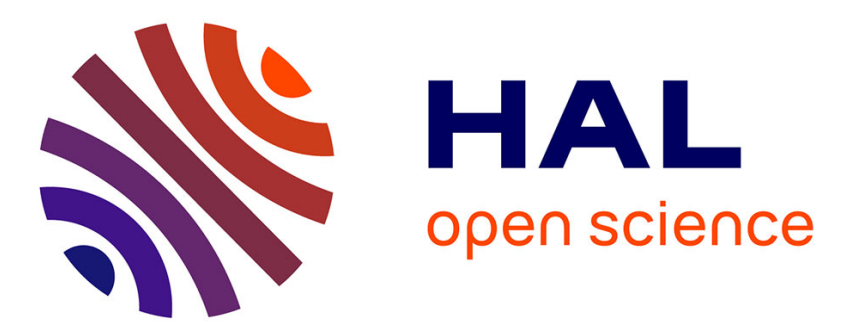

\title{
Event-based controller synthesis by bounding methods
}

Nacim Meslem, Christophe Prieur

\section{To cite this version:}

Nacim Meslem, Christophe Prieur. Event-based controller synthesis by bounding methods. European Journal of Control, 2015, 26, pp.12-21. 10.1016/j.ejcon.2015.09.004 . hal-01235617

\section{HAL Id: hal-01235617 https://hal.science/hal-01235617}

Submitted on 30 Nov 2015

HAL is a multi-disciplinary open access archive for the deposit and dissemination of scientific research documents, whether they are published or not. The documents may come from teaching and research institutions in France or abroad, or from public or private research centers.
L'archive ouverte pluridisciplinaire HAL, est destinée au dépôt et à la diffusion de documents scientifiques de niveau recherche, publiés ou non, émanant des établissements d'enseignement et de recherche français ou étrangers, des laboratoires publics ou privés. 


\title{
Event-based controller synthesis by bounding methods
}

\author{
Nacim Meslem ${ }^{\mathrm{a}}$, Christophe Prieur ${ }^{1 \mathrm{a}}$ \\ ${ }^{a}$ Gipsa-lab, Department of Automatic Control, 11 Rue des Mathématiques, BP 46, 38402 \\ Saint Martin d'Hères, France \\ meslem.nacim@gipsa-lab.fr, christophe.prieur@gipsa-lab.fr
}

\begin{abstract}
Two event-triggered algorithms for digital implementation of a continuoustime stabilizing controller are proposed in this work. The first algorithm updates the control value in order to keep the time evolution of a given Lyapunov-like function framed between two auxiliary functions; whereas the second one actualizes the control value so that the state trajectory of the system stays enclosed between two a priori defined templates. In both cases, a natural hybrid formulation of the event-based stabilizing control problem is used to prove the main results of this work. Furthermore, the existence of a minimum inter-event time greater than zero is proved. Numerical simulations are provided to illustrate the digital implementation of the event-sampling algorithms for nonlinear systems.
\end{abstract}

Keywords: Hybrid systems; Lyapunov methods; global asymptotic stability; event-triggered sampling; reachability analysis

\section{Introduction}

Usually, state feedback control laws applied to dynamical systems are implemented digitally; and the core idea of this discrete-time implementation consists in sampling the continuous-time control law periodically with a sufficiently small sampling period. However, this procedure may be constrained in practice. On the one hand, reducing the sampling period to a level that preserves acceptable performance of the controlled system requires a fairly

\footnotetext{
${ }^{1}$ This work has been partially supported by the LabEx PERSYVAL-Lab (ANR-11LABX- 0025-01).
} 
powerful and so expensive hardware [8]. On the other hand, today's systems are complex and compound by several subsystems controlled by a single CPU. Consequently, reducing the communication between the CPU and the subsystems is a challenge of great interest which allows enhancing the ability to control more complex systems and reducing energy consumption.

To reach this goal, numerous control strategies called event-based approaches have been proposed in the literature, see [14] for a recent framework encompassing the most recent existing event-triggered control techniques. They aim to update the control value only when a significant event occurs. Usually, this event is defined as a deviation threshold on the state vector or on the input vector. In this work, new criteria to design event-triggered sampling algorithms for a large class of nonlinear systems are proposed where the control updating decision is based on the dynamical behavior of auxiliary systems.

The first sampling algorithm updates the control value in order to guarantee that the Lyapunov-like function of the event-based system stays framed at each time instant between the Lyapunov functions of the auxiliary systems. The global stability of the event-controlled system is guaranteed without requiring the ISS stability of each subsystem and satisfying a supplementary small gain condition as needed in [3], where scalar interconnected systems are considered. The second sampling algorithm is based on a component by component comparison of the plant state with a priori defined state templates. In fact, in this case, the control updating procedure aims to force the state trajectory of the event-based system to never leave the state enclosure generated by the auxiliary systems. Moreover, the existence problem of a minimal inter-event time bigger than zero is solved. This algorithm is inspired from the design of event-based controllers by using dead-band methods (see e.g., [9] for an introduction of this method). Consider in particular [13] where only single-input-single-output linear systems are considered. See also recent papers on send-on-delta control techniques dealing with bandlimited signal as in [2].

A preliminary version of this work focused on the case of linear systems has been presented in [11].

The paper is organized as follows. In Section 2 preliminary definitions and notions about hybrid systems, useful to prove our main contributions, are introduced. The problem under consideration is formulated in Section 3 as stability issue of hybrid systems. Sections 4 and 5 state the main contributions of this work regarding the design of event-triggered state feedback 
controls for nonlinear systems. Numerical simulations are provided in Section 6 when focusing on a nonlinear system borrowed from [3]. Section 7 collects concluding remarks.

Notation. In this article the Euclidean inner product of two vectors $x$ and $y$ will be denoted by $x \cdot y$, the induced norm will be denoted by $|\cdot|$. Given a set $\mathcal{A}$, and a point $x,|x|_{\mathcal{A}}$ is the distance of $x$ relative to $\mathcal{A}$, that is $\inf _{z \in \mathcal{A}}|x-z|$. $\operatorname{int} \mathcal{A}$ and $\overline{\mathcal{A}}$ stand respectively for the interior and the closure of $\mathcal{A}$. Given a vector $x$ in $\mathbb{R}^{n}, x^{\top}$ stands for the transpose of $x$. The Lie derivative of a function $V$ with respect to the vector $f$, i.e., $\nabla V \cdot f$ will be denoted by $\mathrm{L}_{f} V$. The inequality operators $\prec, \preceq, \succ$ and $\succeq$ between vectors must be understood component by component, e.g. $x \prec y$ if and only if $x_{i}<y_{i}$ for all $i$ where $x_{i}$ and $y_{i}$ are the $i$ th components of $x$ and $y$ respectively. The $i$-th vector of the canonical basis is denoted by $e_{i}$. A function $\alpha:[0, \infty) \rightarrow \mathbb{R}$ is of class $\mathcal{K}$ if it is zero at zero, continuous and strictly increasing. It is of class $\mathcal{K}_{\infty}$ if it is of class $\mathcal{K}$ and is unbounded. A function $\rho:[0, \infty) \rightarrow \mathbb{R}$ belongs to $\mathcal{P} \mathcal{D}$ (positive definite) if it is continuous, $\rho(s)>0$ for all $s>0$ and zero at zero.

\section{Basic notions on hybrid systems}

This section is devoted to briefly introduce basic definitions and notions on hybrid systems [6] needed to prove the main results of this paper. By definition, hybrid systems are complex dynamical systems that exhibit both continuous and discrete dynamic behavior and viewed as a set of ordinary differential equations (ODE) governed by a finite-state automaton [6]. Mathematically, these dynamical systems can be described as follows

$$
\begin{cases}\dot{x}=f(x), & \text { if } x \in \mathcal{F}, \\ x^{+} \in g(x), & \text { if } x \in \mathcal{J},\end{cases}
$$

where $x \in \mathbb{R}^{n}$ stands for the state of (1) with the vector field $f: \mathbb{R}^{n} \rightarrow \mathbb{R}^{n}$. The set-valued mapping $g: \mathbb{R}^{n} \rightrightarrows \mathbb{R}^{n}$ is the reset function of (1). The sets $\mathcal{F}$ and $\mathcal{J}$ are two closed subsets of $\mathbb{R}^{n}$ respectively called flow and jump sets. Note that, in this work, the design of the two event-triggered sampling algorithms is based on the flow and jump sets. We will define these sets later.

So, the hybrid dynamics involve the notion of compact hybrid time domain (see [6, Definition 2.3]). A set $E$ is a compact hybrid time domain if

$$
E=\bigcup_{j=0}^{J-1}\left(\left[t_{j}, t_{j+1}\right], j\right)
$$


for some finite sequence of times $0=t_{0} \leq t_{1} \ldots \leq t_{J}$. It is a hybrid time domain if for all $(T, J) \in E, E \cap([0, T] \times\{0,1, \ldots J\})$ is a compact hybrid time domain. A solution $x$ to (1) consists of a hybrid time domain dom $x$ and a function $x: \operatorname{dom} x \rightarrow \mathbb{R}^{n}$ such that $x(t, j)$ is absolutely continuous in $t$ for a fixed $j$ and $(t, j) \in \operatorname{dom} x$ satisfying

(S1) for all $j \in \mathbb{N}$ and almost all $t$ such that $(t, j) \in \operatorname{dom} x$,

$$
x(t, j) \in \mathcal{F}, \quad \dot{x}(t, j)=f(x(t, j)),
$$

(S2) for all $(t, j) \in \operatorname{dom} x$ such that $(t, j+1) \in \operatorname{dom} x$,

$$
x(t, j) \in \mathcal{J}, \quad x(t, j+1) \in g(x(t, j)) .
$$

When the state $x(t, j)$ belongs to the intersection of the flow set and of the jump set, then the solution can either flow or jump. Let us emphasize that the state of (1) should be either in $\mathcal{F}$ or in $\mathcal{J}$, and there is no solution issuing from $\mathbb{R}^{n} \backslash(\mathcal{F} \cup \mathcal{J})$.

A solution $x$ to (1) is said to be complete if its domain is unbounded (either in the $t$-direction or in the $j$-direction), Zeno if it is complete but the projection of dom $x$ onto $\mathbb{R}_{\geq 0}$ is bounded, and maximal if there does not exist another solution $\widetilde{x}$ to (1) such that $x$ is a truncation of $\widetilde{x}$ to some proper subset of its domain. Hereafter, only maximal solutions will be considered.

In literature (see e.g. [6, Definition 3.6]), one associates to the hybrid system (1) the following stability definition.

Definition 1. Let $\mathcal{A}$ be a closed subset of $\mathbb{R}^{n}$ and $\mathcal{H}$ be the hybrid system defined in (1). The set $\mathcal{A}$ is said to be

- stable for $\mathcal{H}$ : if for each $\epsilon>0$ there exists $\delta>0$ such that each solution $x$ to $\mathcal{H}$ with $|x(0,0)|_{\mathcal{A}} \leq \delta$ satisfies $|x(t, j)|_{\mathcal{A}} \leq \epsilon$ for all $(t, j) \in \operatorname{dom} x$;

- pre-attractive for $\mathcal{H}$ : if all complete solutions satisfy $\lim _{t+j \rightarrow \infty}|x(t, j)|_{\mathcal{A}}=$ 0 ;

- globally pre-asymptotically stable for $\mathcal{H}$ : if it is both stable and preattractive for $\mathcal{H}$;

- globally asymptotically stable for $\mathcal{H}$ : if it is globally pre-asymptotically stable for $\mathcal{H}$ and if each solution to $\mathcal{H}$ is complete. 


\section{Problem statement}

Consider a nonlinear system

$$
\dot{x}_{p}=f_{p}\left(x_{p}, u\right)
$$

where $f_{p}: \mathbb{R}^{n_{p}} \times \mathbb{R}^{m} \rightarrow \mathbb{R}^{n_{p}}$ is continuously differentiable, $x_{p}$ stands for the state of the plant and $u$ stands for the control.

Assume that there exists a continuous state feedback control law $u=$ $k\left(x_{p}\right)$ for which system (2) in closed loop with $k$ is globally asymptotically stable. Then, the aim of this work is to design event-based sampling algorithms for the stabilizing state feedback control $u=k\left(x_{p}\right)$ by combining reachability analysis with stability analysis of hybrid systems. These sampling algorithms depend on the state of two auxiliary autonomous systems

$$
\begin{aligned}
& \dot{x}_{a}=f_{a}\left(x_{a}\right), \\
& \dot{x}_{b}=f_{b}\left(x_{b}\right),
\end{aligned}
$$

as illustrated by Figure 1. In (3), $f_{a}: \mathbb{R}^{n_{a}} \rightarrow \mathbb{R}^{n_{a}}$ and $f_{b}: \mathbb{R}^{n_{b}} \rightarrow \mathbb{R}^{n_{b}}$ are two continuously differentiable functions.

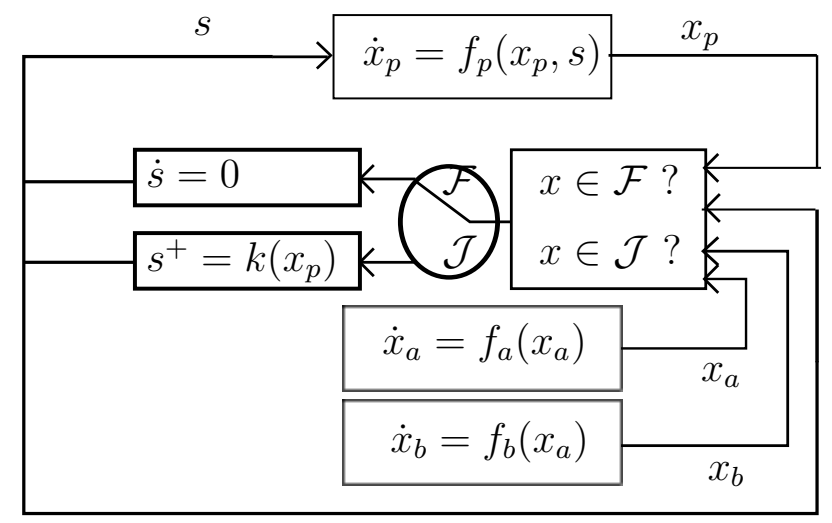

Figure 1: Event-based sampling algorithm for the state feedback controller $k\left(x_{p}\right)$

So, the closed-loop system presented in Figure 1 is more formally written 
as a hybrid system $\mathcal{H}$

$$
\mathcal{H}:\left\{\begin{array}{l}
\dot{x}_{p}=f_{p}\left(x_{p}, s\right) \\
\dot{x}_{a}=f_{a}\left(x_{a}\right) \\
\dot{x}_{b}=f_{b}\left(x_{b}\right) \\
\dot{s}=0 \\
x_{p}^{+} \in\left\{x_{p}\right\} \\
x_{a}^{+} \in k_{a}\left(x_{a}, x_{p}\right) \\
x_{b}^{+} \in k_{b}\left(x_{b}, x_{p}\right) \\
s^{+} \in\left\{k\left(x_{p}\right)\right\}
\end{array} \quad x \in \mathcal{F},\right.
$$

where $x=\left(x_{p}^{\top}, x_{a}^{\top}, x_{b}^{\top}, s^{\top}\right)^{\top}$ in $\mathbb{R}^{n}$ stands for the state of this system, $n=$ $n_{p}+n_{a}+n_{b}+m, k_{a}$ and $k_{b}$ are two set-valued mappings defining the discrete dynamics of $x_{a}$ and $x_{b}$ components, and $\mathcal{F}$ and $\mathcal{J}$ are two closed sets. Roughly speaking, this hybrid implementation is the data of a flow and a jump condition, that define respectively when holding and when updating the control input of system (2). To prove our main results, and to derive these piecewise-constant controller design methods, it is assumed that a Lyapunov function for the closed loop system $\dot{x}_{p}=f_{p}\left(x_{p}, k\left(x_{p}\right)\right.$ ) is available (without any ISS property), see Assumption 1 below for a precise statement of the needed hypothesis.

\section{Comparing values of Lyapunov functions (Algorithm 1)}

The core idea of the first algorithm is to keep the time evolution of a Lyapunov-like function $V_{p}$ of (2) with a piecewise constant input framed between two systems called respectively slow system (with state $V_{s}$ ) and fast system (with state $V_{f}$ ). To achieve this goal, first, this algorithm detects online the time instants when the Lyapunov-like function reaches one of the boundary Lyapunov functions. Then, it updates the control values in order to redirect the Lyapunov-like function towards the inside of the region formed by the slow system with state $V_{s}$ and fast system with state $V_{f}$. This procedure is illustrated in Figure 2.

In this framework, systems (3a) and (3b) will be respectively called slow and fast systems because their Lyapunov functions will bound the Lyapunov function for $(2)$. In the sequel, indices $(a, b)$ are replaced by $(f, s)$ to better fit the context of the present section.

The following assumption will be useful to derive our first main result. 


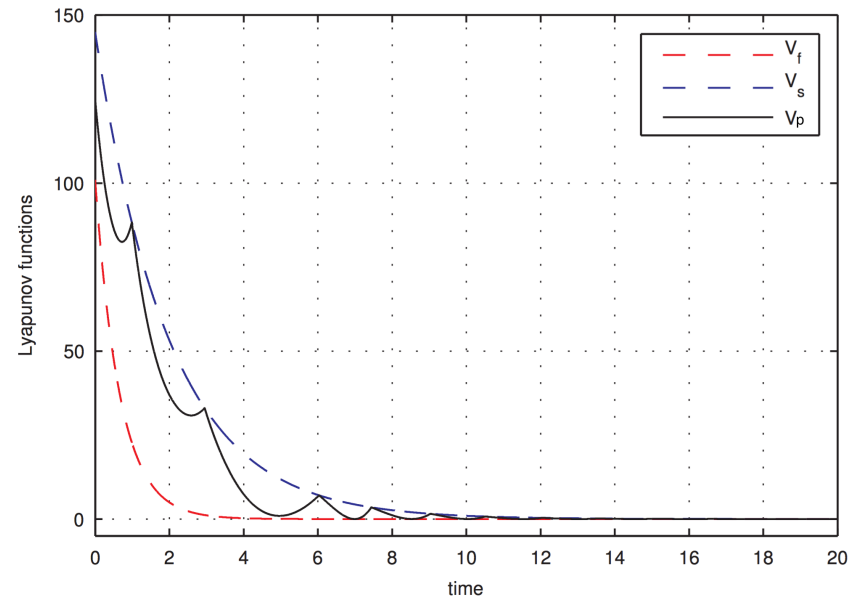

Figure 2: Graphical illustration of the first event-based sampling algorithm.

Assumption 1. There exist an open set $\mathcal{O} \subset \mathbb{R}^{n_{p}+n_{p}+n_{p}}$ containing the origin, a continuous function $k$ : dom $k \rightarrow \mathbb{R}^{m}$, a continuously differentiable function $V_{p}: \operatorname{dom} V_{p} \rightarrow \mathbb{R}$, a function $\rho_{s} \in \mathcal{K}$ such that,

- for all $x_{p} \in \operatorname{dom} k$, it holds

$$
\alpha_{1}\left(\left|x_{p}\right|\right) \leq V_{p}\left(x_{p}\right) \leq \alpha_{2}\left(\left|x_{p}\right|\right)
$$

- $\operatorname{dom} V_{p}=\operatorname{dom} k$ and $\operatorname{dom} V_{p} \times \operatorname{dom} V_{p} \times \operatorname{dom} V_{p}$ contains a neighborhood of $\mathcal{O}$

- for all $\left(x_{p}, x_{f}, x_{s}\right) \in \mathcal{O}$, it holds

$$
\left(x_{p}, x_{p}, x_{p}\right) \in \mathcal{O}, \quad k\left(x_{p}\right) \in \operatorname{dom} k
$$

- for all $\left(x_{p}, x_{f}, x_{s}\right) \in \mathcal{O}, 0 \neq V_{p}\left(x_{f}\right) \leq V_{p}\left(x_{p}\right) \leq V_{p}\left(x_{s}\right)$, it holds

$$
\begin{gathered}
\mathrm{L}_{f_{p}(., k(.))} V_{p}\left(x_{p}\right)<\mathrm{L}_{f_{s}} V_{p}\left(x_{s}\right), \\
\mathrm{L}_{f_{f}} V_{p}\left(x_{f}\right)<\mathrm{L}_{f_{p}(., k(.))} V_{p}\left(x_{p}\right), \\
\quad \mathrm{L}_{f_{s}} V_{p}\left(x_{s}\right) \leq-\rho_{s}\left(\left|x_{s}\right|\right) .
\end{gathered}
$$

Remark 1. For a given closed-loop asymptotically stable nonlinear system $\dot{x}_{p}=f_{p}\left(x_{p}, k\left(x_{p}\right)\right)$, the slow and fast systems can be respectively defined as follows

$$
\begin{aligned}
& \dot{x}_{s}=-\beta_{s} f_{p}\left(x_{s}, k\left(x_{s}\right)\right) \\
& \dot{x}_{f}=-\beta_{f} f_{p}\left(x_{f}, k\left(x_{f}\right)\right)
\end{aligned}
$$

where $\beta_{s} \in(0,1)$ and $\beta_{f}>1$. This approach is employed for the example considered in Section 6 below. 
Remark 2. A stronger assumption than Assumption 1 is when it is assumed moreover that $\mathcal{O}=\mathbb{R}^{n_{p}+n_{p}+n_{p}}$ and when the functions $k$ and $V_{p}$ are defined on all the space. It yields the following more restrictive assumption:

There exist a continuous function $k: \mathbb{R}^{n_{p}} \rightarrow \mathbb{R}^{m}$, a continuously differentiable function $V_{p}: \mathbb{R}^{n_{p}} \rightarrow \mathbb{R}$, two functions $\alpha_{1}$ and $\alpha_{2} \in \mathcal{K}_{\infty}$ and a function $\rho_{s} \in \mathcal{K}$ such that, for all $\left(x_{p}, x_{f}, x_{s}\right) \in \mathbb{R}^{n_{p}+n_{p}+n_{p}}, 0 \neq V_{p}\left(x_{f}\right) \leq V_{p}\left(x_{p}\right) \leq V_{p}\left(x_{s}\right)$, it holds

$$
\begin{gathered}
\mathrm{L}_{f_{p}(., k(.))} V_{p}\left(x_{p}\right)<\mathrm{L}_{f_{s}} V_{p}\left(x_{s}\right), \\
\mathrm{L}_{f_{f}} V_{p}\left(x_{f}\right)<\mathrm{L}_{f_{p}(., k(.))} V_{p}\left(x_{p}\right), \\
\mathrm{L}_{f_{s}} V_{p}\left(x_{s}\right) \leq-\rho_{s}\left(\left|x_{s}\right|\right) .
\end{gathered}
$$

So, the first main result is to find appropriate sets $\mathcal{F}$ and $\mathcal{J}$ so that the attractor is globally asymptotically stable for the hybrid system $\mathcal{H}$. This problem will be solved in Theorem 1 below under Assumption 1, by exploiting Lyapunov methods for hybrid systems (borrowed mainly from [6]).

\subsection{First main result}

Using Assumption 1, it is possible to exploit the comparison of the values of the Lyapunov function $V_{p}$ and to derive a piecewise-constant feedback law for the nonlinear control system (2). This is done in our first main result:

Theorem 1. Under Assumption 1, the set $\{0\} \times\{0\} \times\{0\} \times \mathbb{R}^{m}$ is globally pre-asymptotically stable for the hybrid system $\mathcal{H}$ defined by

$$
\begin{aligned}
& \mathcal{F}=\left\{x \in \mathcal{O} \times \operatorname{dom} k, V_{p}\left(x_{f}\right) \leq V_{p}\left(x_{p}\right) \leq V_{p}\left(x_{s}\right)\right\} \\
& \mathcal{J}=\mathcal{J}_{f} \cup \mathcal{J}_{s} \\
& \mathcal{J}_{f}=\left\{x \in \mathcal{O} \times \operatorname{dom} k,\left\{\begin{array}{c}
V_{p}\left(x_{f}\right)=V_{p}\left(x_{p}\right) \text { and } V_{p}\left(x_{p}\right) \leq V_{p}\left(x_{s}\right) \\
\text { and } \mathrm{L}_{f_{f}} V_{p}\left(x_{f}\right) \geq \mathrm{L}_{f_{p}(., s)} V_{p}\left(x_{p}\right)
\end{array}\right\}\right. \\
& \mathcal{J}_{s}=\left\{x \in \mathcal{O} \times \operatorname{dom} k,\left\{\begin{array}{c}
V_{p}\left(x_{s}\right)=V_{p}\left(x_{p}\right) \text { and } V_{p}\left(x_{p}\right) \geq V_{p}\left(x_{f}\right) \\
\text { and } \mathrm{L}_{f_{s}} V_{p}\left(x_{s}\right) \leq \mathrm{L}_{f_{p}(., s)} V_{p}\left(x_{p}\right)
\end{array}\right\}\right. \\
& k_{f}\left(x_{f}, x_{p}\right)=\left\{x_{p}\right\}, k_{s}\left(x_{s}, x_{p}\right)=\left\{x_{s}\right\}, \forall x \in \mathcal{J}_{f} \\
& k_{f}\left(x_{f}, x_{p}\right)=\left\{x_{f}\right\}, k_{s}\left(x_{s}, x_{p}\right)=\left\{x_{p}\right\}, \forall x \in \mathcal{J}_{s} \\
& k_{f}\left(x_{f}, x_{p}\right)=\left\{x_{p}\right\} \cup\left\{x_{f}\right\}, k_{s}\left(x_{s}, x_{p}\right)=\left\{x_{p}\right\} \cup\left\{x_{f}\right\}, \forall x \in \mathcal{J}_{f} \cap \mathcal{J}_{s}
\end{aligned}
$$

and by (4), with indices $(f, s)$ instead of $(a, b)$.

Before proving this result, let us note that it is quite different to that obtained in [19]. In fact, in [19], it is assumed that the time derivative of the 
Lyapunov function is known at each time instant. In Theorem 1, we relax this assumption by comparing the Lyapunov-like function $V_{p}$ with the Lyapunov functions $V_{s}$ and $V_{f}$ linked to the slow and fast systems, respectively. The proof of the previous result is based on the computation of a weak Lyapunov function and the LaSalle invariance principle is applied. The computation of a strict Lyapunov function is still an open question. It would yield to a simpler proof by applying [6, Theorem 3.18].

Proof. Let us introduce the following function $V: \operatorname{dom} V \rightarrow \mathbb{R}$, defined by, for all $x \in \operatorname{dom} V:=\mathbb{R}^{n_{p}} \times \mathbb{R}^{n_{s}} \times \operatorname{dom} V_{p} \times \mathbb{R}^{m}$,

$$
V(x)=V_{p}\left(x_{s}\right)
$$

and denote

$$
\mathcal{A}=\{0\} \times\{0\} \times\{0\} \times \mathbb{R}^{m} .
$$

Following [6, Definition 3.16], $V$ is a Lyapunov function candidate.

Note that, due to (5) and the expression of $\mathcal{F}$ in Theorem 1, we have, for all $x \in \mathcal{F}$,

$$
\begin{aligned}
\left|x_{f}\right| & \leq \alpha_{1}^{-1}\left(V_{p}\left(x_{f}\right)\right) \leq \alpha_{1}^{-1}\left(V_{p}\left(x_{p}\right)\right) \leq \alpha_{1}^{-1}\left(V_{p}\left(x_{s}\right)\right) \\
& \leq \alpha_{1}^{-1} \alpha_{2}\left(\left|x_{s}\right|\right)
\end{aligned}
$$

and similarly

$$
\left|x_{p}\right| \leq \alpha_{1}^{-1} \alpha_{2}\left(\left|x_{s}\right|\right)
$$

Therefore, for all $x \in \mathcal{F}$,

$$
|x|_{\mathcal{A}}=\left|x_{p}\right|+\left|x_{f}\right|+\left|x_{s}\right| \leq\left(I+2 \alpha_{1}^{-1} \alpha_{2}\right)\left(\left|x_{s}\right|\right)
$$

where $I$ stands for the identity map. Therefore, noting $\left|x_{s}\right| \leq|x|_{\mathcal{A}}$ and using again (5), the definition of $V$ implies that, for all $x$ in $\mathcal{F}$,

$$
V(x) \leq \alpha_{2}\left(\left|x_{s}\right|\right) \leq \alpha_{2}\left(|x|_{\mathcal{A}}\right)
$$

and

$$
V(x) \geq \alpha_{1}\left(\left|x_{s}\right|\right) \geq \alpha_{1}\left(I+2 \alpha_{1}^{-1} \alpha_{2}\right)^{-1}\left(|x|_{\mathcal{A}}\right) .
$$

Let us now introduce the map

$$
G:\left(\begin{array}{c}
x_{p} \\
x_{f} \\
x_{s} \\
s
\end{array}\right) \rightrightarrows\left(\begin{array}{c}
\left\{x_{p}\right\} \\
k_{f}\left(x_{f}, x_{p}\right) \\
k_{s}\left(x_{s}, x_{p}\right) \\
\left\{k\left(x_{p}\right)\right\}
\end{array}\right)
$$


which is the right-hand side of the discrete dynamics of (4). Note that, due to $(6), G(\mathcal{J}) \subset \mathcal{F}$ and observe that $\mathcal{F} \cup \mathcal{J}=\mathcal{F}$. Therefore, defining $\widetilde{\alpha}_{1} \in \mathcal{K}_{\infty}$ by $\widetilde{\alpha}_{1}=\alpha_{1}\left(I+2 \alpha_{1}^{-1} \alpha_{2}\right)^{-1}$, it is deduced from (10)

$$
\widetilde{\alpha}_{1}\left(|x|_{\mathcal{A}}\right) \leq V(x) \leq \alpha_{2}\left(|x|_{\mathcal{A}}\right), \forall x \in \mathcal{F} \cup \mathcal{J} \cup G(\mathcal{J})
$$

Now denote by $F$ the map given by the right-hand side of the continuous dynamics of (4), that is the map

$$
F:\left(\begin{array}{c}
x_{p} \\
s \\
x_{f} \\
x_{s}
\end{array}\right) \mapsto\left(\begin{array}{c}
f_{p}\left(x_{p}, s\right) \\
0 \\
f_{f}\left(x_{f}\right) \\
f_{s}\left(x_{s}\right)
\end{array}\right) .
$$

Due to $(7),(9), \rho_{s} \in \mathcal{K}$, and the expression of $V$, it holds, $\forall x \in \mathcal{F}$,

$$
\mathrm{L}_{F} V(x) \leq-\rho_{s}\left(\left|x_{s}\right|\right) \leq-\rho_{s}\left(I+2 \alpha_{1}^{-1} \alpha_{2}\right)^{-1}\left(|x|_{\mathcal{A}}\right)
$$

Moreover it clearly follows from the expression of $V$ and $G$ that, for all $x \in \mathcal{J}, V(G(x))=V(x)$. Therefore with [6, Proof of the uniform stability in Theorem 3.18] (see also the comment at the beginning of the proof of $[6$, Proposition 3.24]), the closed set $\mathcal{A}$ is stable for the hybrid system $\mathcal{H}$.

To prove that $\mathcal{A}$ is pre-attractive for $\mathcal{H}$ as defined in Definition 1 , let us first prove that each solution to $\mathcal{H}$ is bounded. Due to $L_{F} V(x) \leq 0$, for all $x$ in $\mathcal{F}$, and $V(G(x))=x$, for all $x$ in $\mathcal{J}$, it follows that $V$ is bounded for any solution to $\mathcal{H}$. With $(24)$, it follows that the distance to $\mathcal{A}$ is bounded. Due to the expression of $\mathcal{A}$ in (8), it follows that all components of the solution are bounded, except maybe the last one, that is the $s$-component. Due to the flow and jump dynamics of this component in (4), and since the function $k$ is continuous, the boundedness of the $x_{p}$ component implies the boundedness of the $s$-component. Therefore all solutions to $\mathcal{H}$ are bounded.

To complete the proof of the pre-attractivity, consider now a complete solution to $\mathcal{H}$. The proof of the convergence to $\mathcal{A}$ relies on the invariance principle, see e.g., [18, Theorem 4.3]. To do that, let us first note that that the Standing Assumption of [18, Definition 2.3] is satisfied since the flow and jump sets are closed, and since the dynamics in the hybrid system $\mathcal{H}$ is continuous. Consider a solution $\bar{x}$ to $\mathcal{H}$ which is included in a level set of the function $V$. Let us show that this solution $\bar{x}$ should be in $\mathcal{A}$, i.e. that the $x_{p}, x_{f}$ and $x_{s}$ components of $\bar{x}$ should be 0 . 
Note that for each solution to $\mathcal{H}$ such that it jumps at a given time $(t, j)$ then, either the state is in $\mathcal{A}$ (and then the solution has to stay in $\mathcal{A}$ after time $(t, j))$, or, due to $(7)$ and the expressions of $\mathcal{F}$ and $\mathcal{J}$, the solution has to flow after $(t, j)$.

Due to (13) and since $\rho_{s}\left(I+2 \alpha_{1}^{-1} \alpha_{2}\right)^{-1} \in \mathcal{P} \mathcal{D}$, then the solution $\bar{x}$ cannot flow, except if it is in $\mathcal{A}$. Moreover, it cannot jump, except if it is in $\mathcal{A}$, since, as it has been proven just before, after each jump, either the solution is in $\mathcal{A}$ or it has to flow. Therefore $\bar{x}$ should be in $\mathcal{A}$.

Therefore with [18, Theorem 4.3], any precompact solution to $\mathcal{H}$ approaches $\mathcal{A}$. Moreover, as we have already proven, each complete solution is bounded and thus precompact. Therefore each complete solution to $\mathcal{H}$ approaches $\mathcal{A}$, and $\mathcal{A}$ is pre-attractive for $\mathcal{H}$.

This concludes the proof of Theorem 1.

Remark 3. It is worth pointing out that, from the stability point of view, the event-triggered sampling algorithm can be designed only with the slow Lyapunov function. Here, we have prefered to present the general case where the convergence rate of the event-based system must belong into a desired interval.

\subsection{Existence of a minimum inter-event time}

Let us prove the existence of a (strictly) positive duration between two jump instants for solutions to the hybrid system $\mathcal{H}$ considered in Theorem 1. This property is crucial when implementing this control strategy, since it prevents infinite number of events and infinite number of control updates for digital implementations. This minimal inter-event time is proven in the next result

We are now in position to state the existence of a minimal inter-event time, in the context of Theorem 1, as long as the solution stays outside a neighborhood of the attractor $\mathcal{A}$. To be more precise, we have

Proposition 1. Assume Assumption 1 and $\mathcal{O}=\mathbb{R}^{n_{p}+n_{p}+n_{p}}$ hold. For all $0<r<R$, there exists $t_{\min }>0$ such that for all solutions to $\mathcal{H}$ jumping at two hybrid times $\left(t_{1}, j_{1}\right)<\left(t_{2}, j_{2}\right)$, and satisfying

$$
r \leq|x(t, j)|_{\mathcal{A}} \leq R, \forall\left(t_{1}, j_{1}\right) \leq(t, j) \leq\left(t_{2}, j_{2}\right),
$$

it holds $t_{2}-t_{1} \geq t_{\min }$. 
Proof. Pick $0<r<R$. Due to (10), there exists $\underline{M}$ and $\bar{M}$ such that for all $x$ satisfying $r \leq|x|_{\mathcal{A}} \leq R$, it holds $\underline{M} \leq V(x) \leq \bar{M}$. Let the set $\mathcal{S}=\{x, \underline{M} \leq V(x) \leq \bar{M}\}$. Since $V$ satisfies (12) in the proof of Theorem 1 and since $\widetilde{\alpha}_{1} \in \mathcal{K}_{\infty}$ and $\alpha_{2} \in \mathcal{K}_{\infty}$, the projection of $\mathcal{S}$ in the first $3 n_{p}$ components is compact. Moreover, due to the continuity of the function $k$, due to the fact that the image of a compact set by a continuous function is compact, and due to the expression of the dynamics of the $s$-variable in (4), it follows, on the one hand, that the solutions to $\mathcal{H}$, that are included in $\mathcal{S}$, evolve in a compact set, denoted $K$, whose projection in the $3 n_{p}$ components does not contain the origin.

On the other hand, due to (7) in Assumption 1 and the expressions of the flow and jump sets in Theorem 1, after each jump, each solution is either in $\mathcal{A}$, or it is in the following subset of the flow set described by (7):

$$
\mathcal{F}_{\text {sub }}=\left\{x,\left\{\begin{array}{c}
0 \neq V_{p}\left(x_{f}\right) \leq V_{p}\left(x_{p}\right) \leq V_{p}\left(x_{s}\right) \\
\mathrm{L}_{f_{p}(., k(.))} V_{p}\left(x_{p}\right)<\mathrm{L}_{f_{s}} V_{p}\left(x_{s}\right) \\
\mathrm{L}_{f_{f}} V_{p}\left(x_{f}\right)<\mathrm{L}_{f_{p}(., k(.))} V_{p}\left(x_{p}\right) .
\end{array}\right\}\right.
$$

The set $\mathcal{F}_{\text {sub }}$ has a positive distance to the jump set $\mathcal{J}$ defined in Theorem 1 . Moreover, since the flow dynamics defining $\mathcal{H}$ is defined by a continuous map, and since the set $K$ is compact, we get that all solutions evolving in $K$ have a finite maximal speed $M$. Thus, flowing in $K$ from $\mathcal{F}_{\text {sub }}$ to $\mathcal{J}$ needs a uniform positive time (with a positive lower bound $t_{\min }$ given by the distance between $\mathcal{F}_{\text {sub }}$ to $\mathcal{J}$ over $M$ ). Therefore any solution, evolving in $K$ and having a jump, has to flow for at least a uniform nonzero positive time $t_{\text {min }}>0$. This minimal time between jumps is uniform for all solutions evolving in $K$ and a priori valid only for solutions whose state is in $K$ and thus for all solutions to $\mathcal{H}$ satisfying (14).

This concludes the proof of Proposition 1.

Note that the proof of the previous result has some connections with the proof of [5, Proposition 6], where intermediate (continuous and discrete) time and uniform boundedness of solutions in compact sets are considered.

Since a consequence of Proposition 1 is that all solutions to $\mathcal{H}$ are complete, combining Theorem 1 and Proposition 1, we get the following:

Corollary 1. Under Assumption 1, with $\mathcal{O}=\mathbb{R}^{n_{p}+n_{p}+n_{p}}$, the set $\{0\} \times\{0\} \times$ $\{0\} \times \mathbb{R}^{m}$ is globally asymptotically stable for the hybrid system $\mathcal{H}$ defined in Theorem 1. 
Proof. To prove this corollary, due to Theorem 1, it remains to prove that all solutions are complete.

Let us prove by contradiction that all (maximal) solutions are complete. To do that, let us consider a maximal and incomplete solution $\bar{x}$ to $\mathcal{H}$. Then its hybrid time domain is bounded in the $t$-direction. Therefore due to Proposition 1, for any $0<r<R$, the solution cannot stay in the set $\left\{x, r<|x|_{\mathcal{A}}<R\right\}$. Since each solution to $\mathcal{H}$ is bounded (as proven in the proof of Theorem 1), there exists $\bar{R}>0$ such that the solution lies in $\left\{x,|x|_{\mathcal{A}}<\bar{R}\right\}$. Therefore, we get that for any $r>0$, the solution $\bar{x}$ cannot stay in the set: $\left\{x, r<|x|_{\mathcal{A}}\right\}$. In other words, there exists a sequence of time $\left(t_{n}, j_{n}\right)$ in dom $\bar{x}$ such that, denoting $\bar{x}_{n}=\bar{x}\left(t_{n}, j_{n}\right)$, it holds $\left|\bar{x}_{n}\right|_{\mathcal{A}} \rightarrow 0$, as

$n \rightarrow \infty$. Since the solution $\bar{x}$ is incomplete, the hybrid time domain dom $\bar{x}$ is bounded, and there exists a subsequence (also denoted $\left(t_{n}, j_{n}\right)$ ) and $(T, J)$ in $[0, \infty) \times \mathbb{N}$ such that $\left(t_{n}, j_{n}\right) \rightarrow(T, J)$, as $n \rightarrow \infty$.

Now, using again the boundedness of solutions to $\mathcal{H}$, there exists a subsequence such that $\bar{x}_{n} \rightarrow \bar{x}_{\infty}$ with $\bar{x}_{\infty} \in \mathcal{A}$.

Moreover, if $\bar{x}$ reaches the attractor, then (by the stability property proven in Theorem 1), it remains at the attractor and the solution is also defined for all hybrid times. Therefore, $T$ should be the maximal value of dom $\bar{x}$ in the $t$-direction and $J$ should be the maximal value of dom $\bar{x}$ in the $j$-direction. Now, defining $\widetilde{x}$ as the function equal to $\bar{x}$ for all $(t, j)$ in dom $\bar{x}$ and equal to $\bar{x}_{\infty}$ hereafter, we get a solution to $\mathcal{H}$, and thus a contradiction with the maximality of $\bar{x}$.

Therefore all solutions to $\mathcal{H}$ are complete (maybe there are some Zeno solutions to $\mathcal{H}$ ). This completes the proof of Corollary 1 .

\section{Comparing the state components (Algorithm 2)}

The second event-based sampling algorithm compares the state of the nonlinear control system (2) and the states of two asymptotically stable auxiliary systems. Here these systems are called lower and upper system and their dynamics are described respectively by

$$
\begin{gathered}
\dot{x}_{l}=f_{l}\left(x_{l}\right), \\
\dot{x}_{u}=f_{u}\left(x_{u}\right) .
\end{gathered}
$$

In (15), $f_{l}: \mathbb{R}^{n_{p}} \times \mathbb{R}^{n_{c}} \rightarrow \mathbb{R}^{n_{p}} \times \mathbb{R}^{n_{c}}$ and $f_{u}: \mathbb{R}^{n_{p}} \times \mathbb{R}^{n_{c}} \rightarrow \mathbb{R}^{n_{p}} \times \mathbb{R}^{n_{c}}$ are two continuously differentiable functions, where $n_{c}$ is a positive integer which 
stands for the dimension of an additional state vector which can be used to design the upper and lower systems. Note that, here the indices $(a, b)$ of the hybrid system introduced in (4) are replaced by $(l, u)$. So, at each time instant where the state trajectory of (2) with a piecewise constant input intersects the state trajectories generated by the bounding stable systems (15), the sampling algorithm updates the control value in order to redirect the state trajectory of (2) towards the inside of the region defined by the upper $x_{u}$ and lower $x_{l}$ solutions to (15). This procedure is illustrated in Figure 3
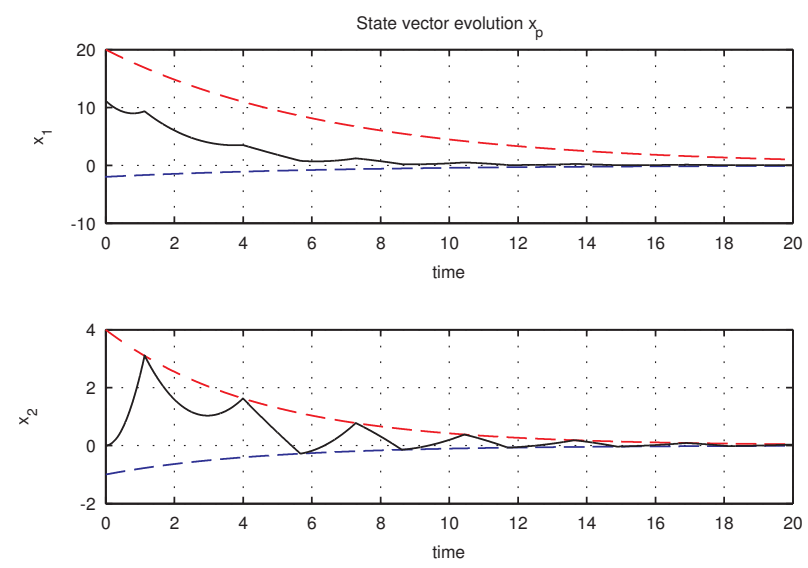

Figure 3: Graphical illustration of the second event-based sampling algorithm.

Before introducing the main assumption needed to prove our results in this context, let us start by defining a projection operator.

Definition 2. Let $\pi: \mathbb{R}^{n_{p}} \times \mathbb{R}^{n_{c}} \rightarrow \mathbb{R}^{n_{p}}$ be the projection operator defined by, for all $\left(x_{p}, x_{c}\right) \in \mathbb{R}^{n_{p}} \times \mathbb{R}^{n_{c}}, \pi\left(x_{p}, x_{c}\right)=x_{p}$.

So, the following assumption will be useful:

Assumption 2. There exist an open set $\mathcal{O} \subset \mathbb{R}^{n_{p}} \times\left(\mathbb{R}^{n_{p}} \times \mathbb{R}^{n_{c}}\right) \times\left(\mathbb{R}^{n_{p}} \times\right.$ $\mathbb{R}^{n_{c}}$ ) containing the origin, a continuous function $k: \operatorname{dom} k \rightarrow \mathbb{R}^{m}$, two continuously differentiable functions $V_{l}: \operatorname{dom} V_{l} \rightarrow \mathbb{R}$ and $V_{u}: \operatorname{dom} V_{u} \rightarrow \mathbb{R}$, two functions $\alpha_{1}$ and $\alpha_{2} \in \mathcal{K}_{\infty}$ and a function $\rho \in \mathcal{K}$ such that

- for all $\left(x_{p}, x_{c}\right) \in \operatorname{dom} V_{l}$, it holds

$$
\alpha_{1}\left(\left|\left(x_{p}, x_{c}\right)\right|\right) \leq V_{l}\left(x_{p}, x_{c}\right) \leq \alpha_{2}\left(\left|\left(x_{p}, x_{c}\right)\right|\right),
$$


and for $\left(x_{p}, x_{c}\right) \in \operatorname{dom} V_{u}$, it holds

$$
\alpha_{1}\left(\left|\left(x_{p}, x_{c}\right)\right|\right) \leq V_{u}\left(x_{p}, x_{c}\right) \leq \alpha_{2}\left(\left|\left(x_{p}, x_{c}\right)\right|\right) ;
$$

- $\operatorname{dom} k \times \operatorname{dom} V_{l} \times \operatorname{dom} V_{u}$ contains a neighborhood of $\mathcal{O}$;

- for all $\left(x_{p}, x_{l}, x_{u}\right) \in \mathcal{O}$, it holds

$$
x_{p}, x_{l}, x_{u} \in \mathcal{O}, \quad k\left(x_{p}\right) \in \operatorname{dom} k ;
$$

- $\operatorname{for}$ all $\left(x_{p}, x_{l}\right) \in \operatorname{dom} k \times \operatorname{dom} V_{l}$ such that $\pi\left(x_{l}\right) \preceq x_{p} \neq 0, \exists i=1, \ldots, n_{p}, \pi\left(x_{l}\right)_{i}=$ $x_{p i}$ and $\pi\left(x_{l}\right) \neq x_{p}$, it holds

$$
\left(\pi\left(f_{l}\left(x_{l}\right)\right)-f_{p}\left(x_{p}, k\left(x_{p}\right)\right)\right) \cdot e_{i}<0
$$

where $e_{i}$ denotes the $i$-th vector of the basis of $\mathbb{R}^{n_{p}}$;

- for all $\left(x_{p}, x_{u}\right) \in \operatorname{dom} k \times \operatorname{dom} V_{u}$ such that $0 \neq x_{p} \preceq \pi\left(x_{u}\right), \exists i=$ $1, \ldots, n_{p}, x_{p i}=\pi\left(x_{u i}\right)$, and $x_{p} \neq \pi\left(x_{u}\right)$, it holds

$$
\left(\pi\left(f_{u}\left(x_{u}\right)\right)-f_{p}\left(x_{p}, k\left(x_{p}\right)\right)\right) \cdot e_{i}>0 ;
$$

- for all $\left(x_{l}, x_{u}\right) \in \operatorname{dom} V_{l} \times \operatorname{dom} V_{u}$, it holds

$$
\begin{aligned}
\mathrm{L}_{f_{l}} V_{l}\left(x_{l}\right) & \leq-\rho\left(\left|x_{l}\right|\right), \\
\mathrm{L}_{f_{u}} V_{u}\left(x_{u}\right) & \leq-\rho\left(\left|x_{u}\right|\right) .
\end{aligned}
$$

This assumption is slightly different to the ones considered in [11], where it is necessary to consider additional functions (compare in particular with [11, Assumption 2]).

Remark 4. This assumption holds for any bounded monotone system (see [20]), where $x_{c}$ is not needed for the upper and lower systems. Some methods to design the lower and upper systems are proposed in $[16,17]$. In addition, for controllable linear systems one can compute a state transformation matrix such that the transformed linear system is monotone with respect to the new state vector (see $[11,15])$. Furthermore, a large class of biological and biotechnological systems satisfies this assumption (see $[21,1,7,4]$ ).

The second main result of this paper is to derive a hybrid implementation of a piecewise constant controller for (2) under Assumption 2. This is done formally in Theorem 2 below by using techniques on hybrid systems. 


\subsection{Second main result}

Our second main result exploits the order preserving properties of dynamical systems [20] so that the state trajectory of the event-based controlled system, described in appropriate coordinates, stays framed between the state trajectories generated by the upper and lower system.

We need a mild technical assumption on the comparison functions considered in Assumption 2:

Assumption 3. The functions $\alpha_{1}, \alpha_{2}, \rho$ in Assumption 2 are such that there exist $\alpha_{3} \in \mathcal{K}_{\infty}$, and three positive values $M_{1}, M_{2}$ and $M_{3}$ satisfying

$$
\begin{gathered}
\alpha_{1}\left(s+s^{\prime}\right) \leq M_{1}\left(\alpha_{1}(s)+\alpha_{1}\left(s^{\prime}\right)\right), \forall s \geq 0, s^{\prime} \geq 0, \\
\alpha_{2}(s)+\alpha_{2}\left(s^{\prime}\right) \leq M_{2} \alpha_{3}\left(s+s^{\prime}\right), \forall s \geq 0, s^{\prime} \geq 0 . \\
\rho\left(s+s^{\prime}\right) \leq M_{3}\left(\rho(s)+\rho\left(s^{\prime}\right)\right), \forall s \geq 0, s^{\prime} \geq 0 .
\end{gathered}
$$

It can be checked that Assumption 3 holds for any linear controllable system selecting $k$ as a stabilizing controller.

Using Assumptions 2 and 3, it is possible to exploit the comparison of the components of the state to derive a piecewise constant controller for the nonlinear control system (2). It yields a hybrid system of the form:

$$
\widetilde{\mathcal{H}}:\left\{\begin{array}{l}
\dot{x}_{p}=f_{p}\left(x_{p}, s\right) \\
\dot{x}_{l}=f_{l}\left(x_{l}\right) \\
\dot{x}_{u}=f_{u}\left(x_{u}\right) \\
\dot{s}=0 \\
x_{p}^{+}=x_{p} \\
x_{l}^{+}=x_{l} \\
x_{u}^{+}=x_{u} \\
s^{+}=k\left(x_{p}\right)
\end{array} \quad \widetilde{x} \in \widetilde{\mathcal{F}},\right.
$$

where $\widetilde{x}=\left(x_{p}^{\top}, x_{l}^{\top}, x_{u}^{\top}, s^{\top}\right)^{\top}$ in $\mathbb{R}^{\widetilde{n}}, \widetilde{n}=n_{p}+\left(n_{p}+n_{c}\right)+\left(n_{p}+n_{c}\right)+m$. This hybrid system is considered in our second main result:

Theorem 2. Under Assumptions 2 and 3, the set $\{0\} \times\{0\} \times\{0\} \times \mathbb{R}^{m}$ is globally pre-asymptotically stable for the hybrid system $\widetilde{\mathcal{H}}$ defined by

$$
\begin{aligned}
& \widetilde{\mathcal{F}}=\left\{\widetilde{x} \in \mathcal{O} \times \operatorname{dom} k, \pi\left(x_{l}\right) \preceq x_{p} \preceq \pi\left(x_{u}\right)\right\} \\
& \tilde{\mathcal{J}}=\left\{\begin{array}{c}
\text { and } \exists i=1, \ldots, n_{p}, \text { such that } \\
\widetilde{x} \in \mathcal{O} \times \operatorname{dom} k,
\end{array}\left\{\begin{array}{c}
\pi\left(x_{l}\right)_{i}=x_{p i} \text { and }\left(\pi\left(f_{l}\left(x_{l}\right)\right)-f_{p}\left(x_{p}, k\left(x_{p}\right)\right)\right) \cdot e_{i} \geq 0 \\
\text { or } \\
\pi\left(x_{u}\right)_{i}=x_{p i} \text { and }\left(\pi\left(f_{u}\left(x_{u}\right)\right)-f_{p}\left(x_{p}, k\left(x_{p}\right)\right)\right) \cdot e_{i} \leq 0
\end{array}\right\}\right.
\end{aligned}
$$


and by (22).

Proof. Let us introduce the following function $\widetilde{V}: \operatorname{dom} \widetilde{V} \rightarrow \mathbb{R}$, defined by, for all $\widetilde{x} \in \operatorname{dom} \widetilde{V}:=\mathbb{R}^{n_{p}} \times \operatorname{dom} V_{l} \times \operatorname{dom} V_{u} \times \mathbb{R}^{m}$,

$$
\widetilde{V}(\widetilde{x})=V_{l}\left(x_{l}\right)+V_{u}\left(x_{u}\right),
$$

and denote $\widetilde{\mathcal{A}}=\{0\} \times\{0\} \times\{0\} \times \mathbb{R}^{m}$. Following [6, Definition 3.16], $\widetilde{V}$ is a Lyapunov function candidate.

Note that, due to the expression of $\widetilde{\mathcal{F}}$ in Theorem 2, we have, for all $\widetilde{x} \in \widetilde{\mathcal{F}}$, and for all $i=1, \ldots, n_{p}$,

$$
\left|x_{p i}\right| \leq \max \left\{\left|x_{l i}\right|,\left|x_{u i}\right|\right\} \leq\left|x_{l i}\right|+\left|x_{u i}\right|,
$$

and thus

$$
\left|x_{p}\right| \leq\left|x_{l}\right|+\left|x_{u}\right|
$$

Therefore, for all $\widetilde{x} \in \widetilde{\mathcal{F}}$,

$$
|\widetilde{x}|_{\widetilde{\mathcal{A}}}=\left|x_{p}\right|+\left|x_{l}\right|+\left|x_{u}\right| \leq 2\left(\left|x_{l}\right|+\left|x_{u}\right|\right)
$$

and, using (16) and (21b), the definition of $\widetilde{V}$ implies that, for all $\widetilde{x}$ in $\widetilde{\mathcal{F}}$,

$$
\begin{aligned}
\widetilde{V}(\widetilde{x}) & \leq \alpha_{2}\left(\left|x_{l}\right|\right)+\alpha_{2}\left(\left|x_{u}\right|\right) \leq M_{2} \alpha_{3}\left(\left|x_{l}\right|+\left|x_{u}\right|\right) \\
& \leq M_{2} \alpha_{3}\left(|\widetilde{x}|_{\widetilde{\mathcal{A}}}\right)
\end{aligned}
$$

and

$$
\begin{aligned}
\widetilde{V}(\widetilde{x}) & \geq \alpha_{1}\left(\left|x_{l}\right|\right)+\alpha_{1}\left(\left|x_{u}\right|\right) \geq M_{1}^{-1} \alpha_{1}\left(\left|x_{l}\right|+\left|x_{u}\right|\right) \\
& \geq M_{1}^{-1} \alpha_{1}\left(\frac{1}{2}|\widetilde{x}|_{\widetilde{\mathcal{A}}}\right) .
\end{aligned}
$$

where (21a) has been used in the second inequality.

Moreover, as in the proof of Theorem 1, using (17), denoting by $G$ the map given by the right-hand side of the discrete dynamics of (22), then it holds

$$
\widetilde{\mathcal{F}} \cup \tilde{\mathcal{J}} \cup G(\tilde{\mathcal{J}})=\tilde{\mathcal{F}}
$$

Therefore, defining $\widetilde{\alpha}_{1}$ and $\widetilde{\alpha}_{2} \in \mathcal{K}_{\infty}$ by $\widetilde{\alpha}_{1}(s)=M_{1}^{-1} \alpha_{1}\left(\frac{1}{2} s\right)$ and $\widetilde{\alpha}_{2}(s)=$ $M_{2} \alpha_{3}(s)$, for all $s \geq 0$, it is deduced from (24)

$$
\widetilde{\alpha}_{1}\left(|\widetilde{x}|_{\widetilde{\mathcal{A}}} \leq \tilde{V}(\widetilde{x}) \leq \widetilde{\alpha}_{2}\left(|\widetilde{x}|_{\widetilde{\mathcal{A}}}\right), \forall \widetilde{x} \in \widetilde{\mathcal{F}} \cup \widetilde{\mathcal{J}} \cup G(\tilde{\mathcal{J}})\right.
$$


Now denote by $F$ the map given by the right-hand side of the continuous dynamics of (22). Due to (20), (21c), (23), $\rho \in \mathcal{K}$, and the expression of $\widetilde{V}$, it holds, for all $\widetilde{x} \in \widetilde{\mathcal{F}}$,

$$
\begin{aligned}
\mathrm{L}_{F} \widetilde{V}(\widetilde{x}) & \leq-\rho\left(\left|x_{l}\right|\right)-\rho\left(\left|x_{u}\right|\right) \leq-M_{3}^{-1} \rho\left(\left|x_{l}\right|+\left|x_{u}\right|\right) \\
& \leq-M_{3}^{-1} \rho\left(\frac{1}{2}|\widetilde{x}|_{\widetilde{\mathcal{A}}}\right) .
\end{aligned}
$$

Therefore, with [6, Proof of the uniform stability in Theorem 3.18], the closed set $\widetilde{\mathcal{A}}$ is stable for the hybrid system $\widetilde{\mathcal{H}}$.

To conclude the proof of Theorem 2 it remains to prove the pre-attractivity. First let us note that all the solutions to $\widetilde{\mathcal{H}}$ are bounded. To do that, from $L_{F} \widetilde{V} \leq 0$, for all $x$ in $\mathcal{F}$, and $\widetilde{V}(G(x)) \leq V(x)$, for all $x$ in $\mathcal{J}$, and from the expression of $\widetilde{\mathcal{A}}$ and (24b), we first deduce (as in the proof of Theorem 1) the boundedness of all components of any solution to $\widetilde{\mathcal{H}}$, except the $s$-component. Then the boundedness of the $s$-component follows from the continuity of the function $k$ and from jump dynamics in (22). The remaining proof of the pre-attractivity relies on the invariance principle.

To apply this invariance property, note that, since the flow and jump sets of $\widetilde{\mathcal{H}}$ are closed and since the dynamics is continuous, it follows that the Standing Assumption of [18, Definition 2.3] is satisfied. Consider a solution $\bar{x}$ to $\widetilde{\mathcal{H}}$ which is included in a level set of the function $\widetilde{V}$. Let us show that this solution $\bar{x}$ should be in $\widetilde{\mathcal{A}}$, i.e. that the $x_{p}, x_{l}$ and $x_{u}$ components of $\bar{x}$ should be 0 .

Due to (25) and since $\rho \in \mathcal{P} \mathcal{D}$, the solution cannot flow, except if it is in $\widetilde{\mathcal{A}}$. Moreover, it cannot jump, except if it is in $\widetilde{\mathcal{A}}$, since, as it has been proven just before, after each jump, either the solution is in $\widetilde{\mathcal{A}}$ or it has to flow. Therefore $\bar{x}$ should be in $\widetilde{\mathcal{A}}$.

Therefore with [18, Theorem 4.3], any precompact solution to $\widetilde{\mathcal{H}}$ approaches $\widetilde{\mathcal{A}}$. Moreover each complete solution that is not in $\mathcal{A}$ is bounded and thus precompact. Therefore each complete solution to $\widetilde{\mathcal{H}}$ approaches $\widetilde{\mathcal{A}}$, and $\widetilde{\mathcal{A}}$ is pre-attractive for $\widetilde{\mathcal{H}}$.

This concludes the proof of Theorem 2 .

Remark 5. Theorems 1 and 2 state two event-triggered algorithms to design, by emulation, event-based controllers. The main difference between the two theorems are the event-triggering conditions. Indeed, Theorem $1 \mathrm{com}-$ pares the norms of the state of three systems (or more precisely the values 
of the Lyapunov function), whereas Theorem 2 exploits the comparison of the states componentwise. It gives different piecewise-constant controllers for $(2)$.

Under the additional assumption $\mathcal{O}=\mathbb{R}^{n_{p}} \times\left(\mathbb{R}^{n_{p}} \times \mathbb{R}^{n_{c}}\right) \times\left(\mathbb{R}^{n_{p}} \times \mathbb{R}^{n_{c}}\right)$, it is possible to prove the existence of a positive minimal inter-event time in the context of Theorem 2. This could be done in a similar way as in Proposition 1. This yields the following result which is similar to Corollary 2.

Corollary 2. Under Assumption 2, with $\mathcal{O}=\mathbb{R}^{n_{p}} \times\left(\mathbb{R}^{n_{p}} \times \mathbb{R}^{n_{c}}\right) \times\left(\mathbb{R}^{n_{p}} \times \mathbb{R}^{n_{c}}\right)$, the set $\{0\} \times\{0\} \times\{0\} \times \mathbb{R}^{m}$ is globally asymptotically stable for the hybrid system $\widetilde{\mathcal{H}}$ defined in Theorem 2.

Proof. To prove this result, assuming $\mathcal{O}=\mathbb{R}^{n_{p}} \times\left(\mathbb{R}^{n_{p}} \times \mathbb{R}^{n_{c}}\right) \times\left(\mathbb{R}^{n_{p}} \times \mathbb{R}^{n_{c}}\right)$, we follow the steps of proof of Proposition 1 and we prove that the solutions enjoy a minimal inter-event time property as long as the state evolves in a given compact set. This yields a completeness property of solutions to $\widetilde{\mathcal{H}}$, and implies with Theorem 2 that the set $\{0\} \times\{0\} \times\{0\} \times \mathbb{R}^{m}$ is globally asymptotically stable for $\widetilde{\mathcal{H}}$.

\section{Illustrative example}

Consider the nonlinear system borrowed from [3]

$$
\left\{\begin{array}{l}
\dot{x}_{p 1}=x_{p 1} x_{p 2}+x_{p 1}^{2} u_{1} \\
\dot{x}_{p 2}=x_{p 1}^{2}+u_{2}
\end{array}\right.
$$

with state variables $\left(x_{p 1}, x_{p 2}\right) \in \mathbb{R}^{2}$ and the input vector $u=\left(u_{1}, u_{2}\right) \in \mathbb{R}^{2}$. This system is stable under the following state-feedback control law [3]

$$
u_{1}\left(x_{p}\right)=-k_{1} x_{p 1} \text { and } u_{2}\left(x_{p}\right)=-k_{2} x_{p 2}
$$

where $k_{1}=16$ and $k_{2}=6$ and admits the following quadratic function as a Lyapunov function

$$
V_{p}\left(x_{p}\right)=\frac{1}{2} x_{p}^{\top} x_{p}
$$

By direct computation one can show that

$$
\dot{V}_{p}\left(x_{p}\right)=-\mu\left(\left|x_{p}\right|\right)=-\left(x_{p 1}^{2}, x_{p 2}\right) Q\left(x_{p 1}^{2}, x_{p 2}\right)^{\top}
$$

where $Q$ is the positive definite matrix $Q=\left[\begin{array}{cc}k_{1} & -1 \\ -1 & k_{2}\end{array}\right]$. Then, it is clear that there exist functions $\alpha_{1}$ and $\alpha_{2} \in \mathcal{K}_{\infty}$, satisfying

$$
\alpha_{1}\left(\left|x_{p}\right|\right) \leq V_{p}\left(x_{p}\right) \leq \alpha_{2}\left(\left|x_{p}\right|\right), \forall x_{p} \in \mathbb{R}^{2} .
$$




\subsection{Applying method from Theorem 1}

First, following Remark 1 , given two positive values such that $\beta_{s} \in(0,1)$ and $\beta_{f}>1$, let us design the "slow" and "fast" systems:

$$
\begin{gathered}
\left\{\begin{array}{l}
\dot{x}_{s 1}=\beta_{s}\left(x_{s 1} x_{s 2}-k_{1} x_{s 1}^{3}\right) \\
\dot{x}_{s 2}=\beta_{s}\left(x_{s 1}^{2}-k_{2} x_{s 2}\right)
\end{array}\right. \\
\left\{\begin{array}{l}
\dot{x}_{f 1}=\beta_{f}\left(x_{f 1} x_{f 2}-k_{1} x_{f 1}^{3}\right) \\
\dot{x}_{f 2}=\beta_{f}\left(x_{f 1}^{2}-k_{2} x_{f 2}\right)
\end{array}\right.
\end{gathered}
$$

Letting $\mathcal{O}=\mathbb{R}^{2} \times \mathbb{R}^{2} \times \mathbb{R}^{2}$, due to (28), we get that Assumption 1 holds and thus, with Corollary 1 , the hybrid system $\mathcal{H}$ is globally asymptotically stable.

Let us compare the solutions of (26) in closed loop with (27) with solutions to the hybrid system $\mathcal{H}$ given by Theorem 1 . More precisely, consider the initial conditions $x_{s}(0)=(6,-5)^{\top}$ for $(30), x_{f}(0)=(2,-1)^{\top}$ for $(31)$, and $x(0)=(4,-3)^{\top}$ for $(26)$ in closed loop with $(27)$ and for $\mathcal{H}$ considered in Theorem 1. The time evolution are given in Figure 4. As shown in this figure, with only 8 control updating time instants, one obtains a similar result to the case of continuous-time control. Moreover, the same example has been considered in [3] where a similar result is obtained with 38 events.

Figure 5 contains a zoom on the time evolution of $V_{p}\left(x_{s}\right), V_{p}\left(x_{f}\right)$ and $V_{p}\left(x_{p}\right)$. It can be noted that, when closing the loop with the piecewise constant control $u$, the Lyapunov-like function of the plant stays always framed between $V_{p}\left(x_{f}\right)$ and $V_{p}\left(x_{p}\right)$.

\subsection{Applying method from Theorem 2}

Before designing the upper and lower systems, note that the sign of the state trajectory of the first state variable of the system (26), in closed loop with (27), is constant and depends on the sign of the initial value. Thus, system (26) under the control law (27) is monotone with respect to the positive orthant $\mathbb{R}_{\geq 0} \times \mathbb{R}_{\geq 0}$ if $x_{p 1}(0) \geq 0$ and monotone with respect to the orthant $\mathbb{R}_{\leq 0} \times \mathbb{R}_{\geq 0}$ if $x_{p 1}(0) \leq 0$. So, for $x_{p 1}(0)$ positive, one can use the following upper and lower systems

$$
\begin{gathered}
\left\{\begin{array}{l}
\dot{x}_{u 1}=x_{u 1} x_{u 2}-k_{1} x_{u 1}^{3} \\
\dot{x}_{u 2}=x_{u 1}^{2}-k_{2} x_{u 2}
\end{array}\right. \\
\left\{\begin{array}{l}
\dot{x}_{l 1}=x_{l 1} x_{l 2}-k_{1} x_{l 1}^{3} \\
\dot{x}_{l 2}=x_{l 1}^{2}-k_{2} x_{l 2}
\end{array}\right.
\end{gathered}
$$



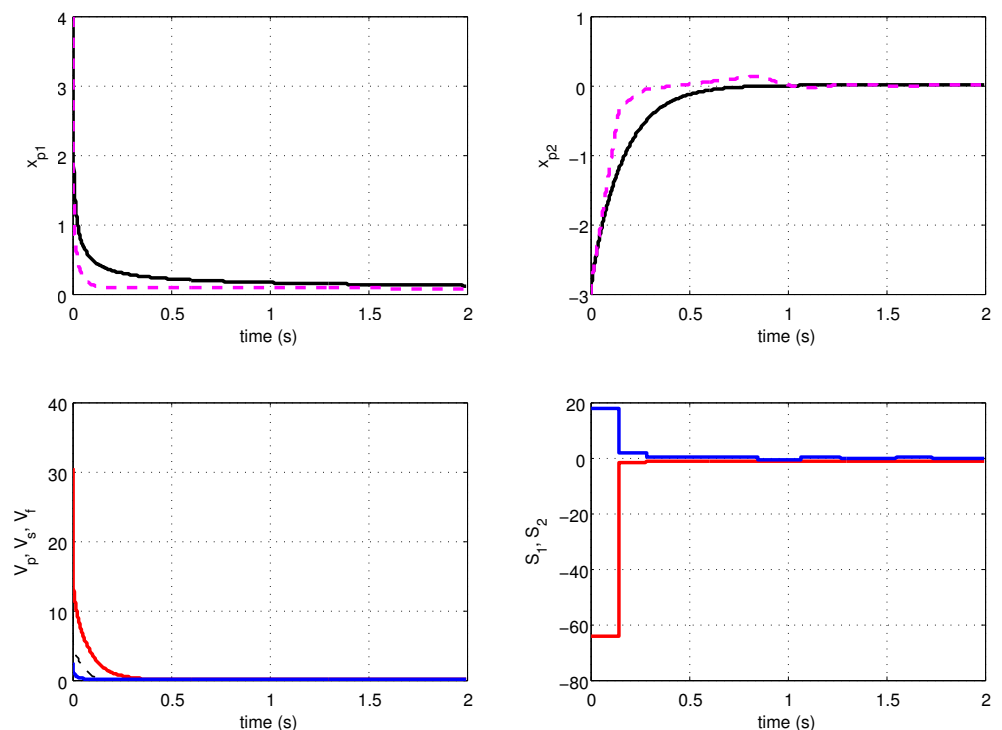

Figure 4: Top pictures show the time evolution of the state variables (dashed lines stand for the event based controlled system and continuous lines stand for the continuous-time controlled system). Bottom left picture shows the time evolution of $V_{p}\left(x_{s}\right), V_{p}\left(x_{f}\right)$ (continuous lines) and $V_{p}\left(x_{p}\right)$ (dashed line). Bottom right picture shows the time evolution of the piecewise constant control law.

and, for negative $x_{p 1}(0)$, one can use the following upper and lower systems

$$
\begin{gathered}
\left\{\begin{array}{l}
\dot{y}_{u 1}=y_{u 1} x_{u 2}-k_{1} y_{u 1}^{3} \\
\dot{x}_{u 2}=y_{u 1}^{2}-k_{2} x_{u 2}
\end{array}\right. \\
\left\{\begin{array}{l}
\dot{y}_{l 1}=y_{l 1} x_{l 2}-k_{1} y_{l 1}^{3} \\
\dot{x}_{l 2}=y_{l 1}^{2}-k_{2} x_{l 2}
\end{array}\right.
\end{gathered}
$$

where $y_{l 1}(0) \in\left[0,-x_{p 1}(0)\right]$ and $y_{u 1}(0) \geq-x_{p 1}(0)$.

Due to (32), (33), (34) and (35), the functions $V_{u}: \mathbb{R}^{2} \rightarrow \mathbb{R}$ and $V_{l}: \mathbb{R}^{2} \rightarrow$ $\mathbb{R}$ can be defined by

$$
V_{u}\left(x_{u}\right)=V_{p}\left(x_{u}\right) \text { and } V_{l}\left(x_{l}\right)=V_{p}\left(x_{l}\right) .
$$

Letting $\mathcal{O}=\mathbb{R}^{2} \times \mathbb{R}^{2} \times \mathbb{R}^{2}$, due to (28) and (36), we get that Assumptions 2 and 3 hold and thus, with Corollary 2, the hybrid system $\mathcal{H}$ is globally asymptotically stable.

Now, let us compare the solutions of (26) in closed loop with (27) with solutions to the hybrid system $\mathcal{H}$ given by Theorem 2. Consider the initial 


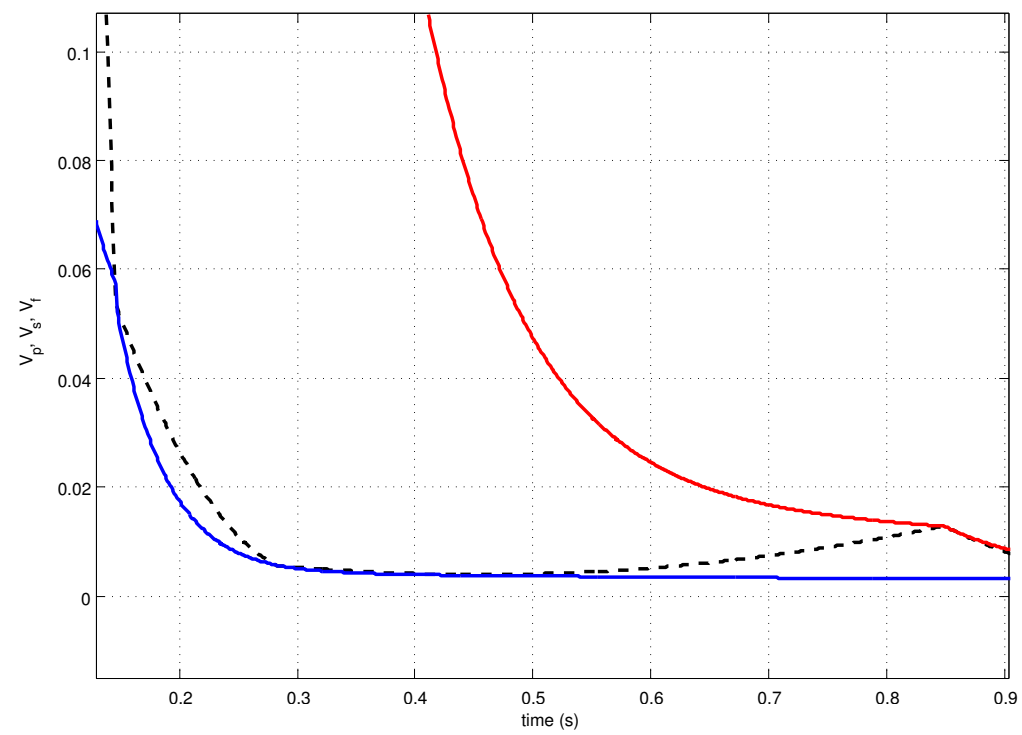

Figure 5: Time evolution of $V_{p}\left(x_{p}\right)$ (dashed black line), $V_{p}\left(x_{s}\right)$ (red line), and $V_{p}\left(x_{f}\right)$ (blue line).

conditions $x_{u}(0)=(8,0)^{\top}$ for $(32), x_{u}(0)=(1,-6)^{\top}$ for $(33)$, and $x_{p}(0)=$ $(4,-3)^{\top}$ for $(26)$ in closed loop with $(27)$ and for $\mathcal{H}$ considered in Theorem 2. The time evolutions are given in Figure 6. As shown in this figure, with only 11 control updating time instants, one obtains a similar result to the case of continuous-time control. Note that, here the components $\left(u_{1}, u_{2}\right)$ of the piecewise control law are updated separately. In fact, we preferred to do that to show the possibility to use our method to deal with the case of distributed control systems [3] where the complex system is composed by scalar subsystems.

Figure 7 shows a zoom on the time evolution of state variables: upper and lower systems (in red and blue bold lines), event-based controlled system (in dashed lines) and continuous-time controlled system (in tiny black lines). So, it can be noted that, when closing the loop with the piecewise constant control $u$, the state trajectories of this system stay always framed between the state trajectories of the upper and lower systems.

\section{Conclusion}

Based on bounding methods for nonlinear systems and stability analysis of hybrid systems, two event-triggered stabilizing controllers have been pro- 

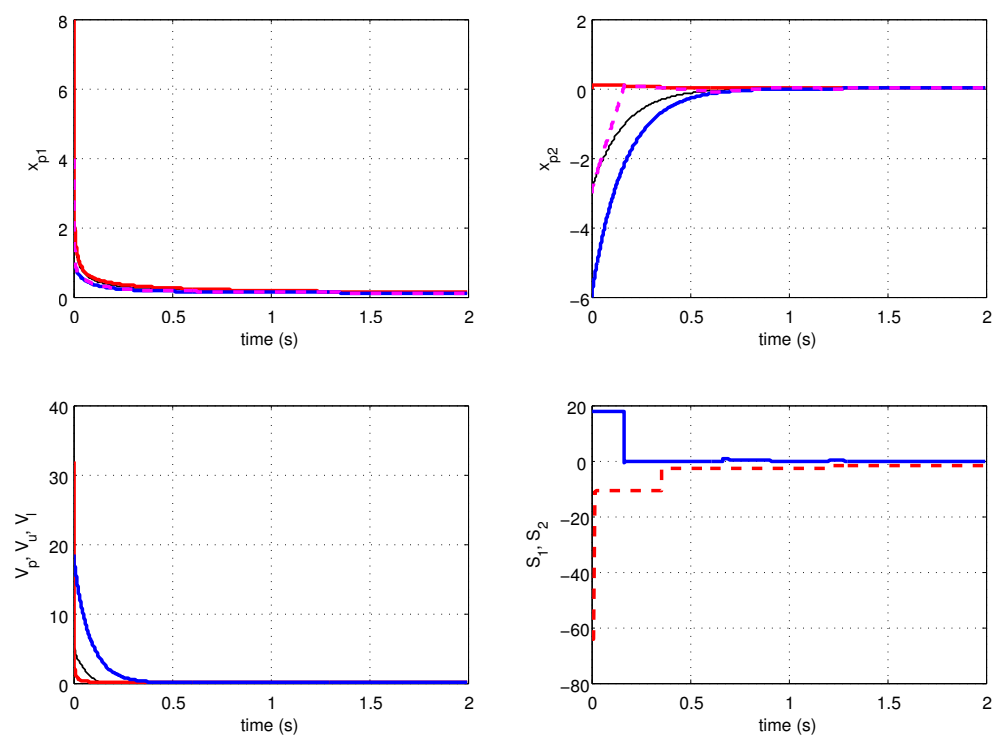

Figure 6: Top pictures show the time evolution of the state variables (dashed lines stand for the event based controlled system and continuous lines stand for the continuous-time controlled system). Bottom left picture shows the time evolution of the Lyapunov functions of the three systems (bold lines stand for the upper and lower systems and the tiny line stands for the event based controlled system). Bottom right picture shows the time evolution of the piecewise constant control law.

posed for nonlinear system for which a static stabilizing controller is given. The control updating strategy is based either on the value of a Lyapunov-like function, or on the comparison of the plant state with given state templates. The obtained stability properties are illustrated by numerical simulations on a nonlinear system. With the first event-based controller, one guarantees that the speed of convergence of the system is included in a given interval formed by the convergence rate of the auxiliary systems (fast and slow); whereas with the second event-based controller, one ensures that the behavior of the system does not violate some specifications defined by a state enclosure.

This paper lets some issues open. In particular, it could be interesting to combine these event-sampling algorithms with interval observer design methods in order to replace, in the control updating criteria, the state vector by its estimate. A preliminary result in that direction has been presented in [12]. It could be also interesting to apply the present work with particular class of nonlinear systems, as the ones with saturating inputs (see e.g. [10] where reset controllers have been computed for such systems). 

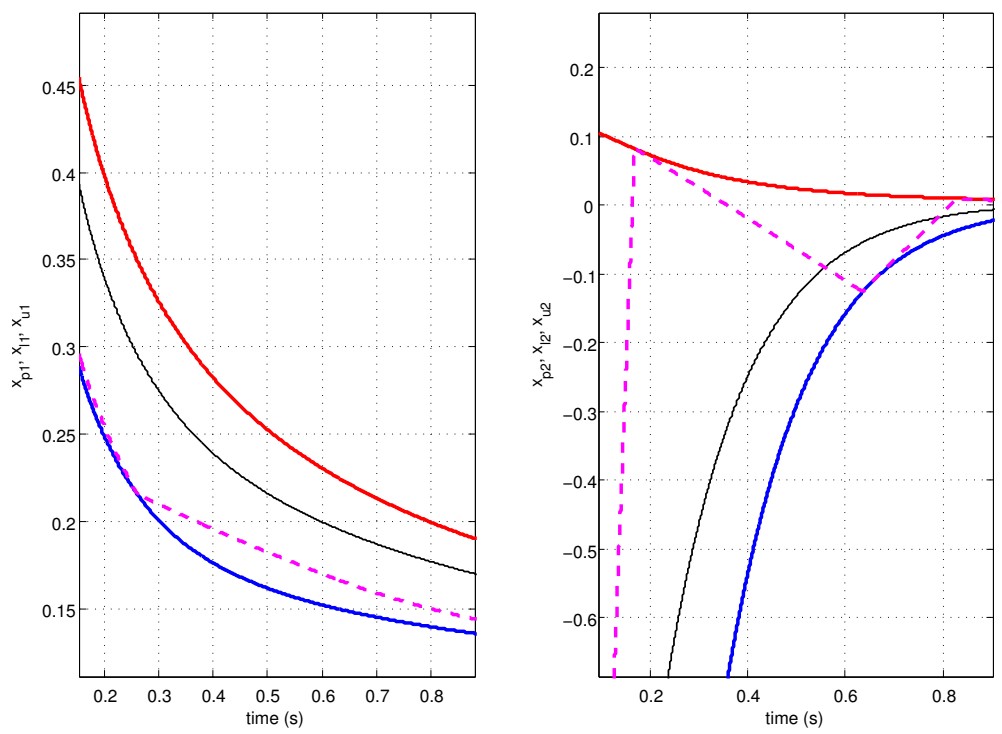

Figure 7: A zoom on time evolution of the state variables. Bold curves stand for the state variables of the upper un lower systems. Tiny curves stand for the state variables of the continuous-time controlled system and the dashed curves show the state variables of the event-based controlled system.

\section{References}

[1] Angeli, D., Sontag, E. D., 2008. Oscillations in I/O monotone systems. IEEE Transactions on Circuits and Systems, Special Issue on Systems Biology 55, 66-176.

[2] Beschi, A., Dormido, S., Sanchez, J., Visioli, A., 2013. Stability analysis of symmetric send-on-delta event-based control systems. In: American Control Conference. Washington (DC), USA, pp. 1771-1776.

[3] De Persis, C., Sailer, R., Wirth, F., 2013. Parsimonious event-triggered distributed control: A zeno free approach. Automatica 49, 2116-2124.

[4] Dochain, D., 2003. State and parameter estimation in chemical and biochemical processes: a tutorial. Journal of Process Control 13 (8), 801-818.

[5] Goebel, R., Sanfelice, R., Teel, A., 2009. Hybrid dynamical systems. IEEE Control Systems Magazine 29 (2), 28-93. 
[6] Goebel, R., Sanfelice, R. G., Teel, A. R., 2012. Hybrid Dynamical Systems: Modeling, Stability, and Robustness. Princeton University Press.

[7] Hadj-Sadok, M., Gouzé, J.-L., 2001. Estimation of uncertain models of activated sludge processes with interval observers. Journal of Process Control 11, 299-310.

[8] Hsu, P., Sastry, S., 1987. The effect of discretized feedback in a closed loop system. In: 26th IEEE Conference on Decision and Control. Los Angeles, California (CA), USA, pp. 1518-1523.

[9] Lehmann, D., 2011. Event-based State-feedback Control. Logos Verlag Berlin.

[10] Loquen, T., Tarbouriech, S., Prieur, C., 2007. Stability analysis for reset systems with input saturation. In: 46th IEEE Conference on Decision and Control (CDC). New Orleans, LA, USA, pp. 3272-3277.

[11] Meslem, N., Prieur, C., 2013. Event-triggered algorithm for continuoustime systems based on reachability analysis. In: 52nd IEEE Conference on Decision and Control. Florence, Italy, pp. 2048-2053.

[12] Meslem, N., Prieur, C., 2015. Event-based stabilizing controller using a state observer. In: 1st IEEE International Conference on Event-based Control, Communication, and Signal Processing. Krakow, Poland.

[13] Pawlowski, A., Cervin, A., Guzman, J., Berenguel, M., 2014. Generalized predictive control with actuator deadband for event-based approaches. IEEE Transactions on Industrial Informatics 10 (1), 523-537.

[14] Postoyan, R., Tabuada, P., Nesic, D., Anta, A., 2015. A framework for the event-triggered stabilization of nonlinear systems. IEEE Transactions on Automatic Control 60 (4), 982 - 996.

[15] Raissi, T., Efimov, D., Zolghadri, A., 2012. Interval state estimation for a class of nonlinear systems. IEEE Transactions on Automatic Control 57 (1), 260-265.

[16] Ramdani, N., Meslem, N., Candau, Y., 2009. A hybrid bounding method for computing an over-approximation for the reachable set of uncertain nonlinear systems. IEEE Transactions on Automatic Control 54 (10), $2352-2364$. 
[17] Ramdani, N., Meslem, N., Candau, Y., 2010. Computing reachable sets for uncertain nonlinear monotone systems. Nonlinear Analysis: Hybrid Systems 4 (2), 263-278.

[18] Sanfelice, R., Goebel, R., Teel, A. R., 2007. Invariance principles for hybrid systems with connections to detectability and asymptotic stability. IEEE Transactions on Automatic Control 52 (12), 2282-2297.

[19] Seuret, A., Prieur, C., Marchand, N., 2014. Stability of non-linear systems by means of event-triggered sampling algorithms. IMA Journal of Mathematical Control and Information 31 (3), 415-433.

[20] Smith, H., 2008. Monotone Dynamical Systems: An Introduction to the Theory of Competitive and Cooperative Systems. Mathematical Surveys and Monographs. American Mathematical Society.

[21] Sontag, E., 2005. Molecular systems biology and control. Eur. J. Control 11(4-5), 396-435. 